\title{
A Robust Optimization of Capacity Allocation Policies in the Third-Party Warehouse
}

\author{
Xu Xian-hao, ${ }^{1}$ Dong Wei-hong, ${ }^{1}$ and Peng Hongxia ${ }^{2}$ \\ ${ }^{1}$ School of Management, Huazhong University of Science and Technology, Wuhan 430074, China \\ ${ }^{2}$ School of Business, Hubei University, Wuhan 430062, China \\ Correspondence should be addressed to Dong Wei-hong; dongweihong@hust.edu.cn
}

Received 10 October 2014; Accepted 15 January 2015

Academic Editor: John Gunnar Carlsson

Copyright (c) $2015 \mathrm{Xu}$ Xian-hao et al. This is an open access article distributed under the Creative Commons Attribution License, which permits unrestricted use, distribution, and reproduction in any medium, provided the original work is properly cited.

\begin{abstract}
We study the capacity allocation policies of a third-party warehouse center, which supplies several different level services on different prices with fixed capacity, on revenue management perspective. For the single period situation, we use three different robust methods, absolute robust, deviation robust, and relative robust method, to maximize the whole revenue. Then we give some numerical examples to verify the practical applicability. For the multiperiod situation, as the demand is uncertain, we propose a stochastic model for the multiperiod revenue management problem of the warehouse. A novel robust optimization technique is applied in this model to maximize the whole revenue. Then we give some numerical examples to verify the practical applicability of our method.
\end{abstract}

\section{Introduction and Literature Review}

In today's business world, a large number of companies outscore their warehouse functions to the third-party Warehouse (3PW) company in order to minimize their operation costs and focus on their core competencies. Therefore, warehousing industry becomes a booming business all over the world. According to the survey data from National Bureau of Statistics (NBS) of China, the national warehousing investment in fixed assets amounted to 69.20 billion dollars in 2013, increasing $32.7 \%$ over 2012. With the fast development of third-party warehousing industry, the revenue problem has received considerable attentions from both 3PW practitioners and researchers. 3PW company can provide storage services to different customers with fixed storage capacity and then capacity allocation policy plays an important role in revenue management.

The aim of capacity allocation in $3 \mathrm{PW}$ is to pursue a better fit between storage capacity allocation and market demand for each level in order to improve the expected revenue. In addition, customer demands for each level are uncertain. In this paper, we focus on the capacity allocation policy of a $3 \mathrm{PW}$ company for both single storage period and multiperiod with a revenue management perspective and robust optimization method.

Revenue management (RM) is a useful tool to help companies sell their products or services to right customers at right price and right time and make greatest revenue [1]. The field of revenue management is originated in the airline industry as a way to efficiently allocate fixed capacity to different classes of customers [2]. Talluri and Van Ryzin [3] discussed the network RM problems extensively, and their work was based on the independent demand model. The success of airline revenue management was widely reported, and this stimulated development of revenue management systems for other transportation sectors and in other areas of the services sectors, such as automobile rental [4], broadcasting [5], and hospitality [6]. Chiang et al. [7] provided an overview paper on revenue management. The solution methods that are mentioned in these papers are mainly mathematical programming, dynamic programming, and heuristics such as genetic algorithm. Revenue management is applied into many industries but not warehouse. In this paper, we study the capacity allocation problem with the perspective on revenue management. 
Several researchers have worked at third-party warehousing. Gong and de Koster [8] gave a review on stochastic models and analysis on warehouse operations. Analysis of thirdparty warehousing contracts with commitments was studied without revenue consideration with capacity allocation in Chen et al. [9]. Lin [10] studied capacity allocation policy of third-party warehouse with dynamic optimization in revenue management perspective. Zhang et al. [11] provided the mathematical model of allocating customers to different warehouse spaces with deterministic demand and they solved this problem with a scheduling approach. Gong et al. [12] proposed a facility design method to improve the warehouse revenue with the consideration of stochastic market demand and the customers arrived according to a Poisson process. However, it is difficult to characterize the distribution of the uncertain demand, and robust optimization with uncertain demand is not included in these papers.

Robust optimization is a useful method to solve stochastic programming with unknown probability. Soyster [13] first proposed factor uncertainty in the field of optimization and gave its robust method. Ben-Tal and Nemirovski [14-16] proposed several uncertainties regarding the form and its applications and analyzed the robust methods of the linear programming and general convex programming. El Ghaoui et al. $[17,18]$ derived a similar conclusion and gave the robust methods to solve optimization problems in practical application. Bertsimas et al. [19, 20] did a further research on the basis of their work; they relaxed the conservation of the robust optimization and introduced the concept of the price of robust. Vairaktarakis [21] presented robust newsboy models with uncertain demand and provided an alternative approach using deterministic optimization models which could be solved by dynamic programming. Their work studied the single period problem, and our paper can solve the capacity allocation problem for both single period and multiple periods.

The rest of this paper is organized as follows. In Section 2, we describe the capacity allocation problem of the 3PW company and the corresponding mathematical model. Then we propose three different robust models of single period problem, the corresponding algorithms, and we give some numerical examples in Section 3. Section 4 presents the robust capacity allocation policies for multiperiod situation and then gives some illustrative examples. Finally, Section 5 concludes the paper with a short summary and future direction.

\section{Problem Description}

In this paper, we consider such a 3PW company which provides several different levels of warehousing service for customers, with fixed capacity $L$. The demand in each level is uncertain. The service price or cost for each storage level is different; therefore the unit revenue for each level is quite different. Facing such condition, this company should decide the storage capacity for each level. As the total capacity is fixed, if we increase one level's capacity, the opportunity cost of other levels maybe occur; if not, satisfaction degree of customer in this level may decrease and give orders to other competitors.
In order to improve the revenue of this warehouse center and use the storage capacity more appropriately, they should provide an appropriate capacity allocation policy which can meet the market demand more accurately. Therefor, this capacity allocation problem is how this 3PW company will allocate its limited warehousing capacity to each storage service level; then it can maximize the total revenue.

Hypotheses are made as the following:

$L:$ total capacity of the 3PW company;

$N$ : number of the service levels;

$L_{n}$ : capacity number of $n$th level, $n=1, \ldots, N$;

$P_{n}$ : the unit price of $n$th level per period;

$C_{n}$ : the unit cost of $n$th level per period, and $C_{n} \leqslant P_{n}$;

$S_{n}$ : the unit cost of lost sales of $n$th level per period;

$D_{n}$ : the demand of $n$th level capacity, it is an interval uncertain variable with the probability density function $f(\cdot)$ and cumulative distribution function $F(\cdot)$;

$\pi_{n}\left(L_{n}, D_{n}\right):$ the revenue of $n$th level capacity with capacity $L_{n}$ and demand $D_{n}$.

The TPW is a unit-load warehouse; that is, all goods in this warehouse need to occupy the same storage space (one pallet); split of the pallet does not exist.

The profit function for the $i$ th item is given by

$\pi_{n}\left(L_{n}, D_{n}\right)= \begin{cases}P_{n} D_{n}-C_{n} L_{n}, & L_{n} \geqslant D_{n} \\ P_{n} L_{n}-\left(C_{n} L_{n}+S_{n}\left(D_{n}-L_{n}\right)\right), & L_{n}<D_{n}\end{cases}$

In a similar way as stochastic knapsack method, dynamic warehousing capacity allocation model is obtained as follows:

$\max$

$$
\begin{aligned}
& E\left(\pi_{n}\left(L_{n}, D_{n}\right)\right) \\
& =\int_{0}^{L_{n}}\left(P_{n} u_{n}-C_{n} L_{n}\right) f\left(u_{n}\right) d u_{n} \\
& \quad+\int_{L_{n}}^{\infty}\left(P_{n} L_{n}-\left(C_{n} L_{n}+S_{n}\left(u_{n}-L_{n}\right)\right)\right) f\left(u_{n}\right) d u_{n}
\end{aligned}
$$

$$
\begin{array}{ll}
\text { s.t. } & \sum_{n=1}^{N} L_{n} \leqslant L \\
& L_{n} \geqslant 0, \quad n=1, \ldots, N .
\end{array}
$$

The first constraint is the total capacity constraint, and the second one implies capacity of each level cannot be negative.

Theorem 1. There exists the optimum solution in formula (2) without constraint. 
Proof. Analyzing formula (2), we can get the first-order derivative as follows:

$$
\begin{aligned}
& \frac{\partial E}{\partial L_{n}=} \frac{\partial}{\partial L_{n}}\left(\int _ { 0 } ^ { L _ { n } } \left(P_{n} u_{n}\right.\right. \\
& \left.\quad-C_{n} L_{n}\right) f\left(u_{n}\right) d u_{n} \\
& \quad+\int_{L_{n}}^{\infty}\left(P_{n} L_{n}-\left(C_{n} L_{n}\right.\right. \\
& \left.\left.\left.\quad+S_{n}\left(u_{n}-L_{n}\right)\right)\right) f\left(u_{n}\right) d u_{n}\right) \\
& =\frac{\partial}{\partial L_{n}}\left(P_{n} \int_{0}^{L_{n}} u_{n} f\left(u_{n}\right) d u_{n}-S_{n} \int_{L_{n}}^{\infty} u_{n} f\left(u_{n}\right) d u_{n}\right. \\
& \left.\quad+\left(P_{n}+S_{n}\right) L_{n}\left(1-F\left(L_{n}\right)\right)-C_{n} L_{n}\right) \\
& =P_{n} L_{n} f\left(L_{n}\right)+S_{n} L_{n} f\left(L_{n}\right)+\left(P_{n}+S_{n}\right)\left(1-F\left(L_{n}\right)\right) \\
& \quad-\left(P_{n}+S_{n}\right) L_{n} f\left(L_{n}\right)-C_{n} \\
& =\left(P_{n}+S_{n}-C_{n}\right)-\left(P_{n}+S_{n}\right) F\left(L_{n}\right) .
\end{aligned}
$$

And the second-order derivative is

$$
\frac{\partial^{2} E}{\partial L_{n}{ }^{2}}=-\left(P_{n}+S_{n}\right) f\left(L_{n}\right) \leqslant 0 .
$$

Now we know that the expected revenue function is a concave function about variable $L_{n}$, so there exists the optimum solution if there is no constraint condition, and it should satisfy the following condition:

$$
F\left(L_{n}^{*}\right)=\frac{P_{n}+S_{n}-C_{n}}{P_{n}+S_{n}} .
$$

However, in practice we can hardly know the cumulative distribution function $F(\cdot)$ of the demand variable $D_{n}$. On the contrary, the maximum and minimum value of $D_{n}$ can easily be got. In the rest of this paper, we discuss the interval demand condition with $D_{n} \in\left[\underline{D_{n}}, \overline{D_{n}}\right]$.

\section{Robust Optimization for Single Period}

There exist many methods to describe the uncertainty in management optimization problems. One of the most classic versions is the assumption that the probability distribution of the random variable is known. However, it is always not realistic in the actual problem. Robust optimization is a useful method to solve stochastic programming with unknown probability.

According to Vairaktarakis [21], there are three different types of robust methods: absolute robust, deviation robust, and relative robust method. Applying these three robust methods in warehousing capacity allocation, we can get the following three models.
3.1. Absolute Robust Model. In case that the demand realizations for item $n$ take values from the interval $D_{n} \in\left[D_{n}, \overline{D_{n}}\right]$, our absolute robust formulation with a budget constraint becomes

$$
\max _{L_{n}} \min _{D_{n} \in\left[\underline{D_{n}}, \overline{D_{n}}\right]} \pi_{n}\left(L_{n}, D_{n}\right) .
$$

Analyzing the objective function, we can get

$$
\begin{aligned}
& \min _{D_{n} \in\left[\underline{D_{n}}, \overline{D_{n}}\right]} \pi_{n}\left(L_{n}, D_{n}\right) \\
& =\min _{D_{n} \in\left[\underline{D_{n}}, \overline{D_{n}}\right]} \begin{cases}P_{n} D_{n}-C_{n} L_{n}, & L_{n} \geqslant L_{n}^{A} \geqslant \underline{D_{n}} \\
P_{n} L_{n} & -\left(C_{n} L_{n}+S_{n}\left(D_{n}-L_{n}\right)\right), \quad L_{n} \leqslant L_{n}^{A} \leqslant \overline{D_{n}}\end{cases} \\
& =\left\{\begin{array}{cl}
P_{n} \underline{D_{n}}-C_{n} L_{n}, & L_{n} \geqslant L_{n}^{A} \geqslant \underline{\underline{D_{n}}} \\
\left(P_{n}+S_{n}-C_{n}\right) L_{n}-S_{n} \overline{D_{n}}, & L_{n} \leqslant L_{n}^{A} \leqslant \overline{D_{n}} .
\end{array}\right.
\end{aligned}
$$

Thus, the absolute robust allocation $L_{n}^{A}$ should satisfy the following equation:

$$
\pi_{n}\left(L_{n}^{A}, \underline{D_{n}}\right)=\pi_{n}\left(L_{n}^{A}, \overline{D_{n}}\right) .
$$

That implies

$$
L_{n}^{A}=\frac{P_{n} \frac{D_{n}}{}+S_{n} \overline{D_{n}}}{P_{n}+S_{n}} .
$$

Now, we can get the absolute robust allocation model with uncertain interval demand as follows:

$$
\begin{array}{ll}
\max _{L_{1}, \ldots, L_{N}} \min _{D_{n} \in\left[\underline{D_{n}}, \overline{D_{n}}\right]} & \sum_{n=1}^{N} \pi_{n}\left(L_{n}, D_{n}\right) \\
\text { s.t. } & \sum_{n=1}^{N} L_{n} \leqslant L .
\end{array}
$$

The following observations can be made for model AR.

Theorem 2. There exists an optimal solution $\left\{L_{1}^{A *}, \ldots, L_{N}^{A *}\right\}$ for this $A R$ model, and $L_{n}^{A *} \in\left[\underline{D_{n}}, L_{n}^{A}\right], n=1, \ldots, N$.

With this theorem, we can get the equivalent form of formula (10):

$$
\max _{L_{1}, \ldots, L_{n}} \sum_{n=1}^{N}\left(\left(P_{n}+S_{n}-C_{n}\right) L_{n}-S_{n} \overline{D_{n}}\right)
$$

The optimal solution of (11) maximizes the quantity $\sum_{n=1}^{N}\left(P_{n}+S_{n}-C_{n}\right) L_{n}$, and therefore AR model can be reduced to a continuous knapsack problem. The corresponding algorithm will be introduced in the next section. 
3.2. Deviation Robust Model. The deviation robust-order quantity is the solution of

$$
\min _{L_{n}} \max _{D_{n} \in\left[\underline{D_{n}}, \overline{D_{n}}\right]}\left(\pi_{n}\left(D_{n}, D_{n}\right)-\pi_{n}\left(L_{n}, D_{n}\right)\right) .
$$

This formulation provides a solution that minimizes over all choices of order quantities the maximum profit loss due to demand uncertainty. This is a mini-max regret approach where the regret is captured by the difference $\pi_{n}\left(D_{n}, D_{n}\right)-$ $\pi_{n}\left(L_{n}, D_{n}\right)$. The profit could be realized if there is no demand uncertainty in which case we would order $L_{n}=D_{n}$.

That equals

$$
\begin{aligned}
& \max _{D_{n} \in\left[\underline{D_{n}}, \overline{D_{n}}\right]}\left(\pi_{n}\left(D_{n}, D_{n}\right)-\pi_{n}\left(L_{n}, D_{n}\right)\right) \\
& =\max _{D_{n} \in\left[\underline{D_{n}}, \overline{D_{n}}\right]}\left\{\begin{array}{l}
\left(P_{n} D_{n}-C_{n} D_{n}\right)-\left(P_{n} D_{n}-C_{n} L_{n}\right), \\
L_{n} \geqslant L_{n}^{D} \geqslant \underline{D_{n}} \\
-\left(\left(P_{n}+S_{n}-C_{n}\right) L_{n}-S_{n} D_{n}\right),
\end{array}\right. \\
& =\max _{D_{n} \in\left[\underline{D_{n}}, \overline{D_{n}}\right]}\left\{\begin{array}{l}
L_{n} \leqslant L_{n}^{D} \leqslant \overline{D_{n}} \\
\left(P_{n}+S_{n}-C_{n}\right) D_{n} \\
-\left(P_{n}+S_{n}-C_{n}\right) L_{n}, \quad L_{n} \leqslant L_{n}^{D} \leqslant \overline{D_{n}}
\end{array}\right. \\
& =\left\{\begin{array}{l}
C_{n} L_{n}-C_{n} \underline{D_{n}}, \quad L_{n} \geqslant L_{n}^{D} \geqslant \underline{D_{n}} \\
\left(P_{n}+S_{n}-C_{n}\right) \overline{D_{n}} \quad D_{n}, \\
-\left(P_{n}+S_{n}-C_{n}\right) L_{n}, \quad L_{n} \leqslant L_{n}^{D} \leqslant \overline{D_{n}} .
\end{array}\right.
\end{aligned}
$$

Thus, the deviation robust allocation should satisfy the following equation:

$$
L_{n}^{D}=\frac{\left(P_{n}+S_{n}-C_{n}\right) \overline{D_{n}}+C_{n} \underline{D_{n}}}{P_{n}+S_{n}} .
$$

Now, we can get the absolute robust allocation model with uncertain interval demand as follows:

$$
\begin{array}{ll}
\min _{L_{1}, \ldots, L_{N}} \max _{D_{n} \in\left[\underline{D_{n}}, \overline{D_{n}}\right]} & \sum_{n=1}^{N}\left(\pi_{n}\left(D_{n}, D_{n}\right)-\pi_{n}\left(L_{n}, D_{n}\right)\right) \\
\text { s.t. } & \sum_{n=1}^{N} L_{n} \leqslant L .
\end{array}
$$

Just as AR model, the objective function equals

$$
\min _{L_{1}, \ldots, L_{n}} \sum_{n=1}^{N}\left(\left(P_{n}+S_{n}-C_{n}\right) \overline{D_{n}}-\left(P_{n}+S_{n}-C_{n}\right) L_{n}\right) .
$$

It makes us maximize $\sum_{n=1}^{N}\left(P_{n}+S_{n}-C_{n}\right) L_{n}$. Therefore DR model reduces to a continuous knapsack problem. The corresponding algorithm will be introduced in the next section.
3.3. Relative Robust Model. The third robust formulation is called relative robustness and the corresponding formulation is given by

$$
\min _{L_{n} D_{n} \in\left[\underline{D_{n}}, \overline{D_{n}}\right]} \frac{\pi_{n}\left(D_{n}, D_{n}\right)-\pi_{n}\left(L_{n}, D_{n}\right)}{\pi_{n}\left(D_{n}, D_{n}\right)}
$$

which minimizes the relative profit loss per unit of profit that could be made if there was no demand uncertainty. Note that the relative profit loss measures the lost profit as a percentage of the profit that could be made if we knew the actual demand.

In the rest of our analysis it will become clear that the three objectives result in very different choices of order quantities. Similar formulations can be written for the case of interval scenarios. The only difference in modeling the continuous case is that there is a constraint $D_{n} \in\left[D_{n}, \overline{D_{n}}\right]$.

As we have analyzed above, it equals

$$
\begin{aligned}
& \max _{D_{n} \in\left[\underline{D_{n}}, \overline{D_{n}}\right]} \frac{\pi_{n}\left(D_{n}, D_{n}\right)-\pi_{n}\left(L_{n}, D_{n}\right)}{\pi_{n}\left(D_{n}, D_{n}\right)} \\
& =\max _{D_{n} \in\left[\underline{D_{n}}, \overline{D_{n}}\right]}\left\{\begin{array}{l}
\frac{\left(P_{n} D_{n}-C_{n} D_{n}\right)-\left(P_{n} D_{n}-C_{n} L_{n}\right)}{P_{n} D_{n}-C_{n} D_{n}} \\
L_{n} \geqslant L_{n}^{R} \geqslant \underline{D_{n}} \\
\left.-\left(\left(P_{n}+S_{n}-C_{n}\right) L_{n}-S_{n} D_{n}\right)\right) \\
\cdot\left(\left(P_{n}+S_{n}-C_{n}\right) D_{n}-S_{n} D_{n}\right)^{-1}, \\
L_{n} \leqslant L_{n}^{R} \leqslant \overline{D_{n}}
\end{array}\right. \\
& =\left\{\begin{array}{l}
\frac{C_{n} L_{n}-C_{n} \underline{D_{n}}}{P_{n} \underline{D_{n}}-C_{n} \overline{D_{n}}}, \overline{D_{n}}-L_{n} \geqslant L_{n}^{R} \geqslant \underline{D_{n}} \\
\frac{\left(P_{n}+S_{n}-\overline{C_{n}}\right) \overline{D_{n}}-\left(P_{n}+S_{n}-C_{n}\right) L_{n}}{P_{n} \overline{D_{n}}-C_{n} \overline{D_{n}}}, \quad L_{n} \leqslant L_{n}^{R} \leqslant \overline{D_{n}} .
\end{array}\right.
\end{aligned}
$$

The last equation has the optimum solution if and only if $L_{n}^{R}=\left(P_{n}+S_{n}\right) \overline{D_{n}} D_{n} /\left(\left(P_{n}+S_{n}-C_{n}\right) D_{n}+C_{n} \overline{D_{n}}\right)$.

Finally, we can get the RR model:

$$
\begin{array}{ll}
\min _{L_{1}, \ldots, L_{N}} \max _{D_{n} \in\left[\underline{D_{n}}, \overline{D_{n}}\right]} \frac{\pi_{n}\left(D_{n}, D_{n}\right)-\pi_{n}\left(L_{n}, D_{n}\right)}{\pi_{n}\left(D_{n}, D_{n}\right)} \\
\text { s.t. } \quad \sum_{n=1}^{N} L_{n} \leqslant L .
\end{array}
$$

That equals

$$
\begin{aligned}
& \min _{L_{1}, \ldots, L_{N}} \sum_{n=1}^{N} \frac{\left(P_{n}+S_{n}-C_{n}\right) \overline{D_{n}}-\left(P_{n}+S_{n}-C_{n}\right) L_{n}}{P_{n} \overline{D_{n}}-C_{n} \overline{D_{n}}} \\
& \quad=\min _{L_{1}, \ldots, L_{N}} \sum_{n=1}^{N} \frac{\left(P_{n}+S_{n}-C_{n}\right) \overline{D_{n}}}{P_{n} \overline{D_{n}}-C_{n} \overline{D_{n}}}-\frac{\left(P_{n}+S_{n}-C_{n}\right)}{P_{n} \overline{D_{n}}-C_{n} \overline{D_{n}}} L_{n} .
\end{aligned}
$$


TABLE 1: Each cost of warehouse $A$.

\begin{tabular}{lccccccc}
\hline Level & $P_{n}$ & $C_{n}$ & $S_{n}$ & $D_{n}$ & $\overline{D_{n}}$ & $P_{n}+S_{n}-C_{n}$ & $\left(P_{n}+S_{n}-C_{n}\right) /\left(P_{n} \overline{D_{n}}-C_{n} \overline{D_{n}}\right)$ \\
\hline 1 & 10 & 4 & 4 & 500 & 700 & 10 & 0.00238 \\
2 & 8 & 2 & 3 & 600 & 700 & 9 & 0.00214 \\
3 & 12 & 5 & 5 & 200 & 300 & 12 & 0.00686 \\
4 & 14 & 4 & 3 & 300 & 450 & 13 & 0.00289 \\
\hline
\end{tabular}

TABLE 2: The capacity allocation policies of 3 types of robust model.

\begin{tabular}{lccccccc}
\hline Level & Prior (A/DR) & Prior (RR) & $L_{n}^{A}$ & $L_{n}^{A *}$ & $L_{n}^{D}$ & $L_{n}^{D *}$ & $L_{n}^{R}$ \\
\hline 1 & 3 & 3 & 557 & 557 & 643 & 643 & 628 \\
2 & 4 & 4 & 627 & 388 & 682 & 171 & 679 \\
3 & 2 & 1 & 229 & 229 & 271 & 271 & 262 \\
4 & 1 & 2 & 326 & 326 & 415 & 415 & 403 \\
\hline
\end{tabular}

3.4. Algorithm and Illustrative Examples. In this section, we adapt the continuous knapsack procedure to the three robust formulations.

\section{Algorithm for AR Model}

Step 1. Define the weight $w_{n}^{A}=P_{n}+S_{n}-C_{n}, n=1, \ldots, N$, and index the items such that $w_{1}^{A} \geqslant w_{2}^{A} \geqslant \cdots \geqslant w_{N}^{A}$.

Step 2. If $L \geqslant \sum_{n=1}^{N} L_{n}^{A}$, then $L_{n}^{A *}=L_{n}^{A}, n=1, \ldots, N$.

Otherwise identify the critical item $t$ such that

$$
t=\underset{i}{\arg \min }\left\{\sum_{j=1}^{N} \underline{D_{j}}+\sum_{j=1}^{i}\left(L_{j}^{A}-\underline{D_{j}}\right) \geqslant L\right\} .
$$

Step 3. If $i<t, L_{i}^{A *}=L_{i}^{A}$; if $i>t, L_{i}^{A *}=\underline{D_{i}}$; if $i=t, L_{i}^{A *}=$ $L-\sum_{j \neq t} L_{j}^{A *}$.

As the algorithm for DR model is the same as the above algorithm, we do not show it again here.

\subsection{Algorithm for RR Model}

Step 1. Define the weight $w_{n}^{R}=\left(P_{n}+S_{n}-C_{n}\right) /\left(P_{n} \overline{D_{n}}-C_{n} \overline{D_{n}}\right)$, $n=1, \ldots, N$, and index the items such that $w_{1}^{R} \geqslant w_{2}^{R} \geqslant \cdots \geqslant$ $w_{N}^{R}$.

Step 2. If $L \geqslant \sum_{n=1}^{N} L_{n}^{R}$, then $L_{n}^{R *}=L_{n}^{R}, n=1, \ldots, N$.

Otherwise identify the critical item $t$ such that

$$
t=\underset{i}{\arg \min }\left\{\sum_{j=1}^{N} \underline{D_{j}}+\sum_{j=1}^{i}\left(L_{j}^{R}-\underline{D_{j}}\right) \geqslant L\right\}
$$

Step 3. For $i<t, L_{i}^{R *}=L_{i}^{R}$; for $i>t, L_{i}^{R *}=\underline{D_{i}}$; for $i=t$, $L_{i}^{R *}=L-\sum_{j \neq t} L_{j}^{R *}$.
3.6. Illustrative Examples. There is a third-party warehouse company $A$, the total capacity is 1500 , and the service price and cost for each level are shown as Table 1. Assume that, for each level $n$, we are given a scenario $D_{n}$ of demand quantities that may be realized. The number of likely minimum and maximum demand of $D_{n}$ is $D_{n}$ and $\overline{D_{n}}$. Then we can calculate the weights $w_{n}^{A} / w_{n}^{D}$ and $w_{n}^{R}$, which are shown in the last two columns.

By Table 1, we can get the prior list of each robust model. Then using the algorithm that we have proposed in last section, we can get the formulation for the three types of robust policies which are shown as Table 2 .

Table 2 indicates that level 4 receives top priority by AR/DR because it results in the highest (profit + lost sale) ratio; level 3 receives top priority by $R R$ as it holds the higher (profit + lost sale) ratio and lower $D$; level 3 is favored by the RR objective while its priority is lower for the AR and DR objectives. Similar conclusions can be made for the remaining items.

As a result, for any budget level and for every one of the three objectives, our formulations result to order the maximum possible number of units starting with high priority items and continue on with items of lower priority. For example, in the relative robust policy, we should first satisfy the first three highest priority levels: level 4, level 3, and level 1 with the capacity allocations 326,529 , and 557 . For level 4 , the only left capacity is 388 . Similarly, we can get the deviation robust policy and relative robust policy, which are shown as the seventh and ninth column in Table 2.

3.7. Analysis of the Three Robust Policies. In this section, we analyze the effect of these three robust policies on the company revenue. In general, we use three scenarios to simulate the demand market: scenario 1 , the lowest situation, the demand of each level is the minimum demand, that is, $500,600,200$, and 300; scenario 2, the highest situation, the demand of each level is the maximum demand, that is, $700,700,300$, and 450; scenario 3 , the middle situation, the demand of each level is $600,650,250$, and 375 . Using the three robust policies in Table 2, we can get the total revenue of the 
TABLE 3: The revenue of each policy.

\begin{tabular}{lcccc}
\hline $\begin{array}{l}\text { Robust } \\
\text { policy }\end{array}$ & Scenario 1 & Scenario 2 & Scenario 3 & Average revenue \\
\hline AR & 8615 & 8298 & 9323 & 8745.3 \\
DR & 5752 & 8866 & 8594 & 7737.3 \\
RR & 6229 & 8776 & 8729 & 7911.3 \\
\hline
\end{tabular}

third-party warehouse company under each robust policy in Table 3.

From Table 3, we can get that, for scenario 1 (the minimum demand situation), the best policy is absolute robust policy, and the best revenue is 8615 , while the worst revenue of the deviation robust policy is 5752. For the maximum demand situation, the best policy is the deviation robust policy, and the best revenue is 8866 , while the worst revenue of the absolute robust policy is 8298 . For the middle demand situation, the best revenue (9323) comes from the absolute robust policy, while the worst revenue (8594) comes from the deviation robust policy. And we can express Table 3 by Figure 1.

By Figure 1, we can observe that, in both the minimum and middle demand situation, the absolute robust policy is better than the relative robust policy, while the relative robust policy is better than deviation robust policy. However, the opposite result appears in the middle demand situation. From the average revenue, the best policy is absolute robust policy while the worst policy is the deviation robust policy.

The results make sense for the $3 \mathrm{PW}$ company holder. On one hand, if the demand market is not so high, that is, the demand for each level is lower, he should choose the absolute robust policy and avoid deviation robust policy. Otherwise, he should choose deviation robust or relative robust policy when the demand for each level is high. On the other hand, from the perspective of average revenue, the absolute robust policy is the best policy for the conservative holders, whose managements are risk aversion.

\section{Robust Optimization for Multiple Periods}

In this section, we extend this capacity allocation problem into multiperiod condition. The following are the new hypotheses which are used in this section, and the remaining parameters are the same as last section.

$D_{i j n}$ : storage demand which starts from $i$ th period and ends at $j$ th period of $n$th service level $(0 \leq i<j \leq T)$, and it is an uncertain variable;

$r_{n}$ : the unit revenue of storage service level $n$;

$L_{i j n}$ : decision variables, the capacity allocation which starts from $i$ th period and ends at $j$ th period of $n$th service level;

$\sum_{j=i+1}^{T} L_{i j n}$ : the number of products of $n$th service level which are stored on the $i$ th period;

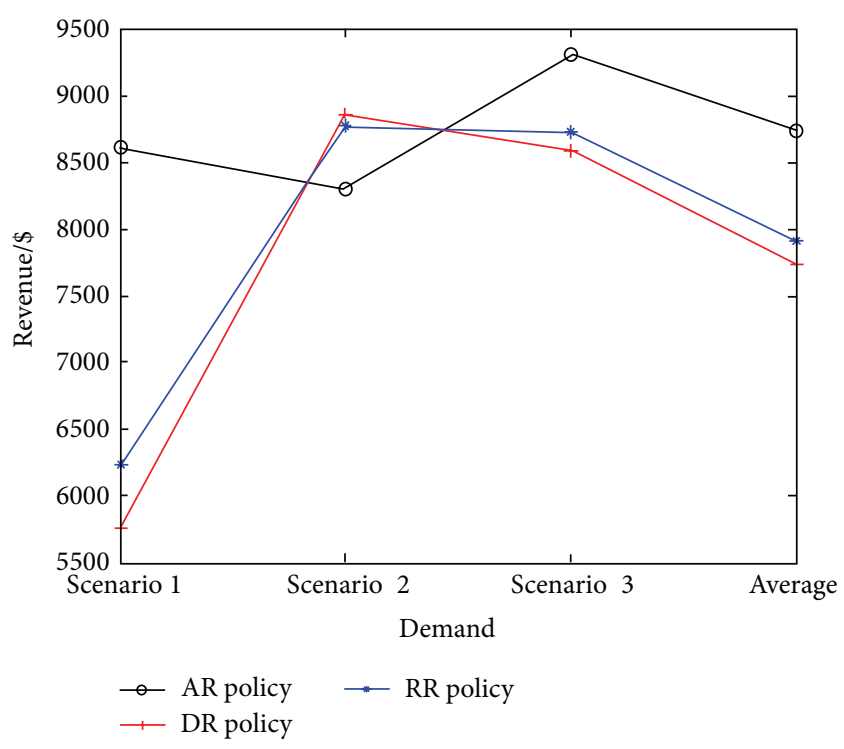

FIGURE 1: The revenue of each policy under each scenario.

$\sum_{i=0}^{j-1} L_{i j n}$ : the number of products of $n$th service level which are retrieved on the $j$ th period.

We assume there are no goods staying before period 0 and all the goods have to be retrieved on or before the last period. The 3PW is a unit-load warehouse; that is, all goods in this warehouse need to occupy the same storage space (one pallet); split of the pallet does not exist.

We consider a particular period $t, t=1,2, \ldots, T-1$; the following equation models the occupation status of the warehouse center on period $t$ :

$$
\sum_{i=0}^{t-1} \sum_{j=t}^{T} L_{i j n}+\sum_{j=t+1}^{T} L_{t j n}-\sum_{i=0}^{t-1} L_{i t n} .
$$

The first part of this equation stands for the number of goods which stay over period $t$, that is, storage before period $t$ and retrieve after period $t$; the second part means the number of goods which are stored on period $t$; the last part means the number of the goods which retrieve on period $t$.

With the fixed capacity, we have the following constraints for period $t$ :

$$
\sum_{i=0}^{t-1} \sum_{j=t}^{T} L_{i j n}+\sum_{j=t+1}^{T} L_{t j n}-\sum_{i=0}^{t-1} L_{i t n} \leq L_{n}
$$

Particularly, on the period 0, we have

$$
\sum_{j=1}^{T} L_{0 j n} \leq L_{n}, \quad n=1,2, \ldots, N
$$


Then we can get the stochastic mathematical model as follows:

$$
\begin{array}{ll}
\max & \sum_{n=1}^{N} \sum_{i=0}^{T-1} \sum_{j=i+1}^{T} r_{n} \cdot(j-i) L_{i j n} \\
\text { s.t. } \quad & \sum_{i=0}^{t-1} \sum_{j=t}^{T} L_{i j n}+\sum_{j=t+1}^{T} L_{t j n}-\sum_{i=0}^{t-1} L_{i t n} \leq L_{n}, \\
& t=1,2, \ldots, T-1, \quad n=1,2, \ldots, N ; \\
& \sum_{j=1}^{T} L_{0 j n} \leq L_{n}, \quad n=1,2, \ldots, N ; \\
& \sum_{n=1}^{N} L_{n} \leq L ; \\
& L_{i j n} \leq D_{i j n}, \quad D_{i j n} \in N \cup\{0\}, \\
& n=1,2, \ldots, N, \quad 0 \leq i<j \leq T .
\end{array}
$$

The objective function is to maximize the total revenue of all period and all service level. The third constraint condition is the total capacity constraint; it means that in every time period the sum of capacity allocations of all service level can not be larger than the total capacity of the 3PW. The last constraint condition stands for the fact that the capacity allocation variable $L_{i j n}$ must be an integer which is not smaller than 0 .

4.1. Robust Optimization Model. The problem looks like a linear integer programming problem. Unfortunately, the parameters $D_{i j n}$ are usually uncertain at the beginning of planning period. Moreover, the revenues may not be fixed, as the decision maker would like to set different pricing, which in turn results in different demands. One may want to solve this by replacing the parameters by their best point estimator, for instance, using expected value $E\left[D_{i j n}\right]$ to replace the uncertain parameter of $D_{i j n}$. One of the most classic versions is the assumption that the probability distribution of the random variable is known. However, it is always not realistic in the actual problem. Robust optimization is a useful method to solve stochastic programming with unknown probability. We believe decision makers would prefer to use proactive tools to obtain their solutions.

According to Mulvey et al. [22], there are two definitions about robustness.

Definition 3 (solution robustness). An optimal solution is solution robust with respect to optimality if it remains "close" to being optimal for any scenario $s \in \theta$.

Definition 4 (model robustness). An optimal solution is model robust with respect to feasibility if it remains "almost" feasible for any scenario $s \in \theta$.
Consider such a stochastic programming:

$$
\begin{array}{ll}
\min & c^{T} x+d^{T} y \\
\text { s.t. } & A x=b \\
& B x+C y=0 \\
& x \geq 0, \quad y \geq 0,
\end{array}
$$

where the decision variable $y$ contains uncertainty. Then the corresponding robust model can be written as follows:

$$
\begin{array}{ll}
\min & \sigma\left(x, y_{1}, \ldots, y_{s}\right)+w \rho\left(\delta_{1}, \delta_{2}, \ldots, \delta_{s}\right) \\
\text { s.t. } & A x=b \\
& B_{s} x+C_{s} y_{s}+\delta_{s}=e_{s}, \quad s \in \theta \\
& x \geq 0, \quad y_{s} \geq 0, \quad \delta_{s} \geq 0, \quad s \in \theta,
\end{array}
$$

where $\delta_{s}$ is the deviation associated with the corresponding scenario $s$. In the objective function of this model, the first part stands for the measure of the solution robustness, and the second part means deviation from the constraint, that is, the measure of the model robustness.

There are several different forms of $\sigma$. In this paper, we use a form of $\sigma$ which was proposed by $\mathrm{Yu}$ and $\mathrm{Li}$ [23]:

$$
\sigma=\sum_{s \in \theta} p_{s} \cdot \xi_{s}+\lambda \sum_{s \in \theta} p_{s} \cdot\left|\xi_{s}-\sum_{s \in \theta} p_{s} \cdot \xi_{s}\right|,
$$

where $p_{s}$ is the probability of the scenario $s$ and $\sum_{s \in \theta} p_{s}=1$.

Under this form and the above robust model, we can get a robust formulation of model (26):

$$
\begin{array}{ll}
\max & \sum_{s \in \theta} p_{s} \cdot \pi_{s}-\lambda \sum_{s \in \theta} p_{s} \cdot\left|\pi_{s}-\sum_{s \in \theta} p_{s} \cdot \pi_{s}\right| \\
& -\sum_{s \in \theta} p_{s} \sum_{n=1}^{N} \sum_{i=0}^{T-1} \sum_{j=i+1}^{T} w_{i j n}\left|D_{i j n}^{s}-L_{i j n}\right| \\
\text { s.t. } \quad & \sum_{i=0}^{t-1} \sum_{j=t}^{T} L_{i j n}+\sum_{j=t+1}^{T} L_{t j n}-\sum_{i=0}^{t-1} L_{i t n} \leq L_{n}, \\
& t=1,2, \ldots, T-1, \quad n=1,2, \ldots, N ; \\
& \sum_{j=1}^{T} L_{0 j n} \leq L_{n}, \quad n=1,2, \ldots, N ; \\
& \sum_{n=1}^{N} L_{n} \leq L ; \\
& L_{i j n} \leq \max \left\{D_{i j n}^{s}\right\}, \\
& n=1,2, \ldots, n, \quad 0 \leq i<j \leq T, \quad s \in \theta \\
& L_{i j n} \in N \cup\{0\}, \quad n=1,2, \ldots, N, 0 \leq i<j \leq T ; \\
& \pi_{s}=\sum_{n=1}^{N} \sum_{i=0}^{T-1} \sum_{n=1}^{N} r_{n}^{s} \cdot(j-i) L_{i j n}, \quad s \in \theta \\
& \lambda \geq 0, \quad w_{i j n} \geq 0,
\end{array}
$$


where $\lambda$ and $w_{i j n}$ are nonnegative weighting parameters. In the objective function of this model (30), the first part is the expected revenue, while the second term is the mean absolute deviation of the revenue. We can regard parameter $\lambda$ as a risk trade-off factor between expected revenue and deviation. The absolute deviation in the third term is a model robustness measurement while the parameters $w_{i j n}$ are the penalty weights for the constraints violations.

In model (30), there is uncertain variable $D_{i j n}$ in both objective function and constraints. In this paper, we use factor-based demand model. See and Sim [24] proposed a form of uncertain variable which can be written as this: $D_{i j n} \triangleq$ $D_{i j n}^{0}+\sum_{k=1}^{K} D_{i j n}^{k} z_{k}$, where $z \triangleq\left\{z_{1}, \ldots, z_{K}\right\}$, and the demand for each level is affinely dependent on uncertain factor $z_{k}$ : $k=1, \ldots, K ; K$ represents the number of such factors used to model demand. With the assumption $U$ and theorems in Ang et al. [25], we can get the equivalent form of the constraint $D_{i j n}^{s}-L_{i j n}+\delta_{i j n}^{s} \geq 0$ as follows:

$$
\begin{array}{ll}
\max & \sum_{k=1}^{K} D_{i j n}^{s, k} z_{k} \\
\text { s.t. } & \sum_{k=1}^{K}\left(\bar{D}_{i j n}^{s, k} \alpha_{k, 1}+D_{i j n}^{s, k} \beta_{k, 1}\right) \geq L_{i j n}-D_{i j n}^{s, 0}-\delta_{i j n}^{s}, \\
& n=1,2, \ldots, N, 0 \leq i<j \leq T \\
& \alpha_{k, 1} \geq 0, \quad \beta_{k, 1} \geq 0, \quad k=1, \ldots, K .
\end{array}
$$

We can use a similar method to deal with the other constraint containing $D_{i j n}$. Finally, model (30) can be transformed in a linear term as follows:

$$
\begin{array}{r}
\max \sum_{s \in \theta} p_{s} \pi_{s}-\lambda \sum_{s \in \theta} p_{s}\left(\pi_{s}-\sum_{s \in \theta} p_{s} \pi_{s}+2 \varepsilon^{s}\right) \\
-\sum_{s \in \theta} p_{s} \sum_{i=0}^{T-1} \sum_{j=i+1}^{T} \sum_{n=1}^{N} w_{i j n} \\
\cdot\left(D_{i j n}^{s, 0}+\sum_{k=1}^{K} D_{i j n}^{s, k} z_{k}\right. \\
\left.-L_{i j n}+2 \delta_{i j n}^{s}\right)
\end{array}
$$

$$
\begin{array}{ll}
\text { s.t. } & \pi_{s}-\sum_{s \in \theta} p_{s} \cdot \pi_{s}+\varepsilon^{s} \geq 0 \\
& \sum_{k=1}^{K}\left(\bar{D}_{i j n}^{s, k} \alpha_{k, 1}+D_{i j n}^{s, k} \beta_{k, 1}\right) \geq L_{i j n}-D_{i j n}^{s, 0}-\delta_{i j n}^{s}, \\
n=1,2, \ldots, N, \quad 0 \leq i<j \leq T \\
\sum_{i=0}^{t-1} \sum_{j=t}^{T} L_{i j k}+\sum_{j=t+1}^{T} L_{t j n}-\sum_{i=0}^{t-1} L_{i t n} \leq C_{k}, \\
t=1,2, \ldots, T-1, \quad n=1,2, \ldots, N ;
\end{array}
$$

$$
\begin{aligned}
& \sum_{j=1}^{T} L_{0 j n} \leq L_{n}, \quad n=1,2, \ldots, N ; \\
& \sum_{n=1}^{N} L_{n} \leq L ; \\
& \sum_{k=1}^{K}\left(\bar{D}_{i j n}^{s, k} \alpha_{k, 2}+D_{i j n}^{s, k} \beta_{k, 2}\right) \geq L_{i j n}-D_{i j n}^{s, 0}, \\
& \quad n=1,2, \ldots, N, 0 \leq i<j \leq T \\
& \pi_{s}=\sum_{n=1}^{N} \sum_{i=0}^{T-1} \sum_{n=1}^{N} r_{n}^{s} \cdot(j-i) L_{i j n}, \quad s \in \theta \\
& L_{i j n} \in N \cup\{0\}, \quad n=1,2, \ldots, N, 0 \leq i<j \leq T \\
& \lambda, w_{i j n}, \varepsilon^{s}, \delta_{i j n}^{s} \geq 0, \\
& n=1,2, \ldots, N, \quad 0 \leq i<j \leq T, \quad s \in \theta \\
& \alpha_{k, 1}, \alpha_{k, 2}, \beta_{k, 1}, \beta_{k, 2} \geq 0, \quad k=1, \ldots, K .
\end{aligned}
$$

The prominent feature of formulation (32) is that it is now in a linear programming form and ready to be solved by popular linear modeling packages like LINGO when the weighting parameters are assigned by the decision makers.

4.2. Illustrative Examples and Analysis. Consider such a $3 \mathrm{PW}$ company which can provide three different levels of storage service for the storage customers. According to the history data, there are three main demand scenarios $s_{1}, s_{2}$, and $s_{3}$; each demand scenario stands for a market condition. For example, $s_{1}$ may mean the market demand is high, and the storage demand of the three levels is large. $s_{2}$ means the storage market is bad, and the demand of each storage level is small. $s_{3}$ is the common condition, and the storage demand is medium. Suppose the probability of these three scenarios is $0.2,0.2$, and 0.6 . The planning horizon is set to be 3 periods, and the total capacity of this warehouse is 2000 units. Demands for all pairs are forecast as shown in Tables 4-6.

In these tables, the "ST" means the storage time and "RT" means the retrieval time of the goods. The first number in Table 4 means that the demand which is stored in period 0 and retrieve in period 1 is 120 . Suppose that, under multiple demand scenarios, the unit price of level 1 for each scenario is 20,15 , and 18 dollars per period, the unit price of level 2 is 30 , 26 , and 28 dollars per period, and the unit price of level 3 is 38,32 , and 36 dollars per period. For simplicity, all weights $w_{i j n}$ are set to be equal to 1 . By our robust model (32), we can get the optimal capacity allocation policies summarized in Table 7.

The total revenue of the $3 \mathrm{PW}$ is 219357 dollars. According to solution the linear programming model, the capacity allocation for level 1 is 951 units, the capacity allocation for level 2 is 612 units, and the capacity allocation for level 3 is 437 units. The optimal capacity allocation policies are summarized in Table 7. 
TABle 4: Demands of customers (scenario $s_{1}$ ).

\begin{tabular}{lcccccccccccc}
\hline \multirow{2}{*}{ ST: $i$} & \multicolumn{3}{c}{$\mathrm{RT}: j($ level 1) } & \multicolumn{4}{c}{$\mathrm{RT}: j$ (level 2) } & \multicolumn{4}{c}{ RT: $j$ (level 3) } \\
& 1 & 2 & 3 & 4 & 1 & 2 & 3 & 4 & 1 & 2 \\
\hline 0 & 200 & 180 & 270 & 150 & 120 & 130 & 190 & 140 & 100 & 180 & 70 \\
1 & 0 & 350 & 280 & 120 & 0 & 200 & 150 & 135 & 0 & 95 & 110 & 50 \\
2 & 0 & 0 & 280 & 100 & 0 & 0 & 160 & 150 & 0 & 0 & 130 & 230 \\
3 & 0 & 0 & 0 & 200 & 0 & 0 & 0 & 120 & 0 & 0 & 0 \\
\hline
\end{tabular}

TABLE 5: Demands of customers (scenario $s_{2}$ ).

\begin{tabular}{lcccccccccccc}
\hline \multirow{2}{*}{ ST: $i$} & \multicolumn{3}{c}{$\mathrm{RT}: j$ (level 1) } & \multicolumn{4}{c}{$\mathrm{RT}: j$ (level 2) } & \multicolumn{4}{c}{ RT: $j$ (level 3) } \\
& 1 & 2 & 3 & 4 & 1 & 2 & 3 & 4 & 1 & 2 \\
\hline 0 & 100 & 140 & 290 & 100 & 90 & 100 & 120 & 110 & 120 & 100 & 70 \\
1 & 0 & 320 & 180 & 120 & 0 & 150 & 200 & 120 & 0 & 80 & 100 & 40 \\
2 & 0 & 0 & 220 & 90 & 0 & 0 & 120 & 100 & 0 & 0 & 100 & 150 \\
3 & 0 & 0 & 0 & 150 & 0 & 0 & 0 & 100 & 0 & 0 & 0 \\
\hline
\end{tabular}

TABLE 6: Demands of customers (scenario $s_{3}$ ).

\begin{tabular}{lcccccccccccc}
\hline \multirow{2}{*}{$\mathrm{ST}: i$} & \multicolumn{3}{c}{$\mathrm{RT}: j($ level 1) } & \multicolumn{4}{c}{$\mathrm{RT}: j$ (level 2) } & \multicolumn{4}{c}{$\mathrm{RT}: j$ (level 3) } \\
& 1 & 2 & 3 & 4 & 1 & 2 & 3 & 4 & 1 & 2 \\
\hline 0 & 130 & 200 & 250 & 120 & 100 & 120 & 170 & 120 & 90 & 150 & 90 \\
1 & 0 & 270 & 310 & 100 & 0 & 180 & 180 & 150 & 0 & 100 & 75 \\
2 & 0 & 0 & 240 & 120 & 0 & 0 & 150 & 120 & 0 & 0 & 110 & 200 \\
3 & 0 & 0 & 0 & 180 & 0 & 0 & 0 & 120 & 0 & 0 & 0 \\
\hline
\end{tabular}

TABLE 7: Capacity allocation policy with multiple demands.

\begin{tabular}{|c|c|c|c|c|c|c|c|c|c|c|c|c|}
\hline \multirow{2}{*}{ ST: $i$} & \multicolumn{4}{|c|}{ RT: $j$ (level 1) } & \multicolumn{4}{|c|}{ RT: $j$ (level 2) } & \multicolumn{4}{|c|}{ RT: $j$ (level 3) } \\
\hline & 1 & 2 & 3 & 4 & 1 & 2 & 3 & 4 & 1 & 2 & 3 & 4 \\
\hline 0 & 170 & 180 & 270 & 110 & 120 & 120 & 185 & 125 & 120 & 80 & 90 & 50 \\
\hline 1 & 0 & 270 & 250 & 100 & 0 & 195 & 180 & 140 & 0 & 120 & 110 & 50 \\
\hline 2 & 0 & 0 & 200 & 110 & 0 & 0 & 150 & 135 & 0 & 0 & 130 & 230 \\
\hline 3 & 0 & 0 & 0 & 200 & 0 & 0 & 0 & 120 & 0 & 0 & 0 & 70 \\
\hline
\end{tabular}

According to Table 7, we can obverse that the demand of level 3 should be met firstly. That is because the unit revenue of level 3 is the highest; therefore the capacity allocation for level 3 can meet all the demand of level 3 for all scenarios. On the contrary, capacity allocation for level 1 is smaller than its market demand as a result of its lower unit revenue. 3TW company managers can improve their revenue by applying this optimization method.

\section{Conclusions and Future Directions}

In this paper, we consider the capacity allocation problem in 3PW company which provides several different level storage services in different price under uncertain market demand. On the revenue management perspective, we propose the mathematical formulations of this problem for both single and multiple periods condition. For the single period situation, as the demand is uncertain, we use three robust methods, absolute robust, deviation robust, and relative robust, to maximize the whole revenue. Based on the analysis of the optimal solution in each situation, we adapt continuous knapsack method to give the corresponding algorithm. Then we use some numerical examples to verify the practical applicability of our method. And we find that the 3PW company managers should provide the maximum possible units of the storage service level with high priority. As the objective function of each method is different, these three methods do not perform the same under the same market scenario. We find that the absolute robust method performs better than the other two methods in most situations. For the multiperiod situation, we propose a stochastic model for the multiperiod revenue management problem of the warehouse. A novel robust optimization technique is applied in this model to maximize the whole revenue. Then we give some numerical examples to verify the practical applicability of our method. The major contribution of this paper is that we use robust optimization to deal with the uncertainty of market demand in 3PW industry. In many of existing references of 3PW revenue management, authors consider revenue optimization under deterministic demand or suppose stochastic demand with known distribution such as Poisson process. In this paper, we do not know the distribution of market demand 
in $3 \mathrm{PW}$ industry and linearize the uncertain mathematical programming by different robust methods.

There remain several limitations in our work. First, we consider the demand of each level and each period is independent. Actually, the demands between different levels may affect each other in some conditions and the demand in one period may be affected by its demand in last period. In our future research, we can analyze the affecting factors of demand and characterize the form of demand to improve the match degree between capacity allocation and demand. Secondly, we set the price of each level as exogenous variables in this paper. In the following research, we can combine the dynamic pricing policy and capacity allocation to improve the revenue of $3 \mathrm{PW}$ more efficiently.

\section{Conflict of Interests}

The authors declare that there is no conflict of interests regarding the publication of this paper.

\section{Acknowledgments}

The authors would like to thank the Associate Editor and anonymous referees for their constructive comments and suggestions, which have led to significant improvements of the paper. This research is partially supported by National Natural Science Foundation of China (nos. 71131004, 71471071) and Humanities and Social Sciences Foundation of Chinese Ministry of Education (no. 12YJC630149).

\section{References}

[1] J. I. McGill and G. J. van Ryzin, "Revenue management: research overview and prospects," Transportation Science, vol. 33, no. 2, pp. 233-256, 1999.

[2] C. P. Wright, H. Groenevelt, and R. A. Shumsky, "Dynamic revenue management in airline alliances," Transportation Science, vol. 44, no. 1, pp. 15-37, 2010.

[3] K. T. Talluri and G. J. Van Ryzin, The Theory Anpractice of Revenue Management, Kluwer Academic Publishers, Boston, Mass, USA, 2004

[4] M. K. Geraghty and E. Johnson, "Revenue management saves National Car Rental,” Interfaces, vol. 27, no. 1, pp. 107-127, 1997.

[5] R. G. Cross, Revenue Management, Broadway Books, Bantam, Doubleday, Dell Publishing Group, New York, NY, USA, 1998.

[6] G. R. Bitran and S. M. Gilbert, "Managing hotel reservations with uncertain arrivals," Operations Research, vol. 44, no. 1, pp. 35-49, 1996.

[7] W. C. Chiang, J. C. Chen, and X. Xu, "An overview of research on revenue management: current issues and future research," International Journal of Revenue Management, vol. 1, no. 1, pp. 97-128, 2007.

[8] Y. Gong and R. B. M. de Koster, "A review on stochastic models and analysis of warehouse operations," Logistics Research, vol. 3, no. 4, pp. 191-205, 2011.

[9] F. Y. Chen, S. H. Hum, and J. Sun, "Analysis of third-party warehousing contracts with commitments," European Journal of Operational Research, vol. 131, no. 3, pp. 603-610, 2001.
[10] C. Lin, "Capacity allocation policy of third party warehousing with dynamic optimization," in Proceedings of the 5th International Symposium on Advances in Computation and Intelligence (ISICA '10), vol. 6382 of Lecture Notes in Computer Science, pp. 297-303, Springer, Wuhan, China, 2010.

[11] X. Zhang, Y. Gong, and S. van de Velde, "Storage scheduling decision models for revenue management of self-storage warehouses," in Proceedings of the 10th International TRAIL in Perspective Congress, 2008.

[12] Y. Gong, R. B. M. de Koster, J. B. G. Frenk, and A. F. Gabor, "Increasing the revenue of self-storage warehouses by facility design," Production and Operations Management, vol. 22, no. 3, pp. 555-570, 2013.

[13] A. L. Soyster, "Technical note-convex programming with setinclusive constraints and applications to inexact linear programming," Operations Research, vol. 21, no. 5, pp. 1154-1157, 1973.

[14] A. Ben-Tal and A. Nemirovski, "Robust convex optimization," Mathematics of Operations Research, vol. 23, no. 4, pp. 769-805, 1998.

[15] A. Ben-Tal and A. Nemirovski, "Robust solutions of linear programming problems contaminated with uncertain data," Mathematical Programming, vol. 88, no. 3, pp. 411-424, 2000.

[16] A. Ben-Tal and A. Nemirovski, "On polyhedral approximations of the second-order cone," Mathematics of Operations Research, vol. 26, no. 2, pp. 193-205, 2001.

[17] L. El Ghaoui and H. Lebret, "Robust solutions to least-squares problems with uncertain data," SIAM Journal on Matrix Analysis and Applications, vol. 18, no. 4, pp. 1035-1064, 1997.

[18] L. El Ghaoui, F. Oustry, and H. Lebret, "Robust solutions to uncertain semidefinite programs," SIAM Journal on Optimization, vol. 9, no. 1, pp. 33-52, 1999.

[19] D. Bertsimas, D. Pachamanova, and M. Sim, "Robust linear optimization under general norms," Operations Research Letters, vol. 32, no. 6, pp. 510-516, 2004.

[20] D. Bertsimas and M. Sim, “The price of robustness," Operations Research, vol. 52, no. 1, pp. 35-53, 2004.

[21] G. L. Vairaktarakis, "Robust multi-item newsboy models with a budget constraint," International Journal of Production Economics, vol. 66, no. 3, pp. 213-226, 2000.

[22] J. M. Mulvey, R. J. Vanderbei, and S. A. Zenios, "Robust optimization of large-scale systems," Operations Research, vol. 43, no. 2, pp. 264-281, 1995.

[23] C. S. Yu and H. L. Li, "A robust optimization model for stochastic logistic problems," International Journal of Production Economics, vol. 64, no. 1, pp. 385-397, 2000.

[24] C.-T. See and M. Sim, "Robust approximation to multiperiod inventory management," Operations Research, vol. 58, no. 3, pp. 583-594, 2010.

[25] M. Ang, Y. F. Lim, and M. Sim, "Robust storage assignment in unit-load warehouses," Management Science, vol. 58, no. 11, pp. 2114-2130, 2012. 


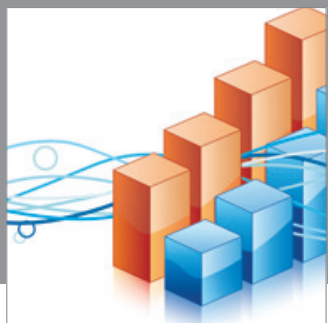

Advances in

Operations Research

mansans

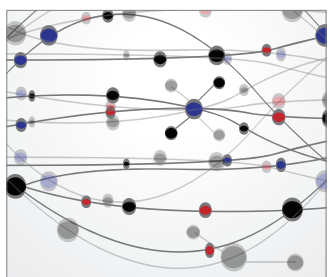

The Scientific World Journal
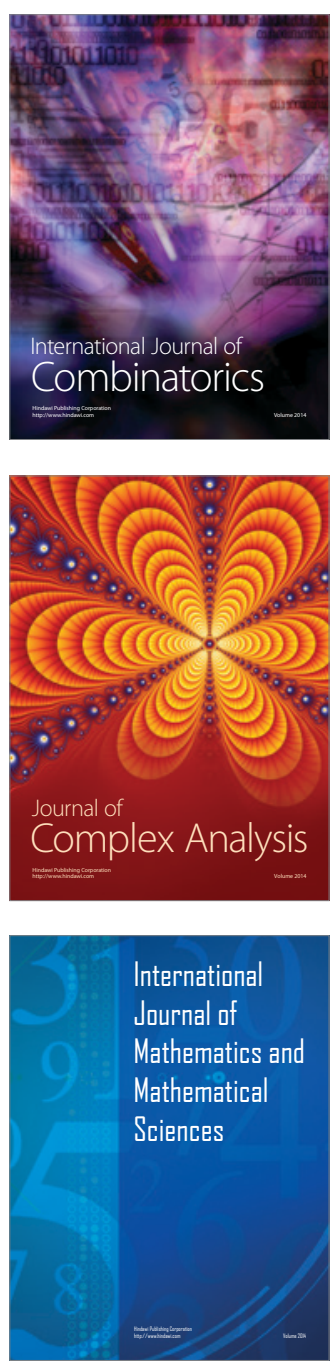
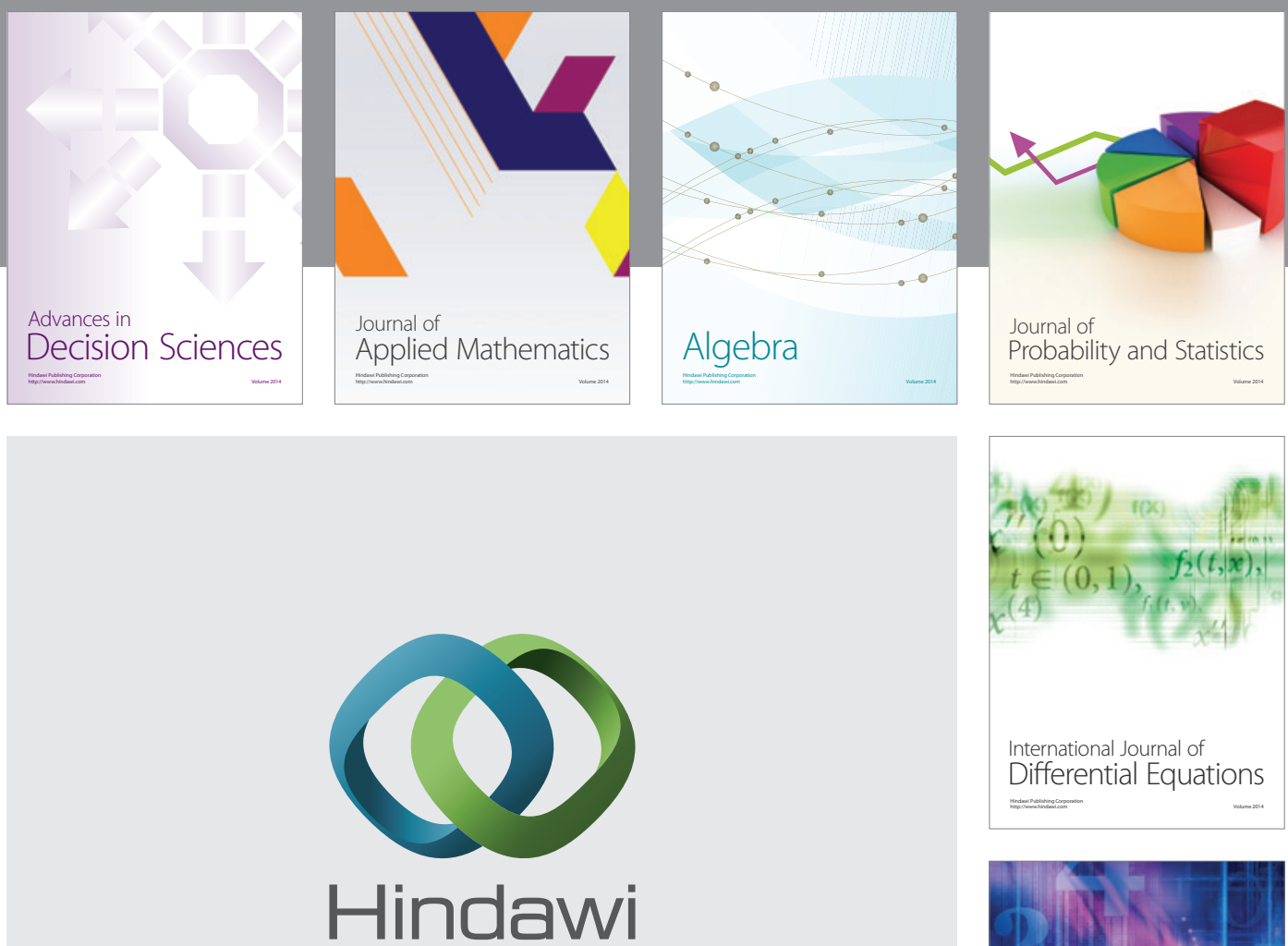

Submit your manuscripts at http://www.hindawi.com


Journal of

Function Spaces

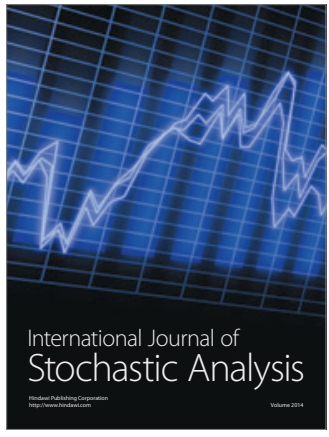

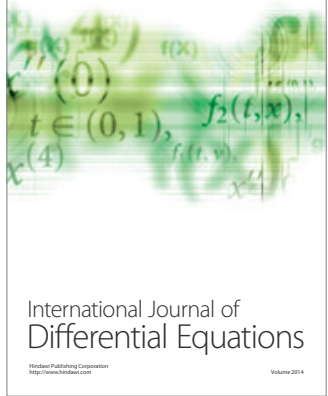
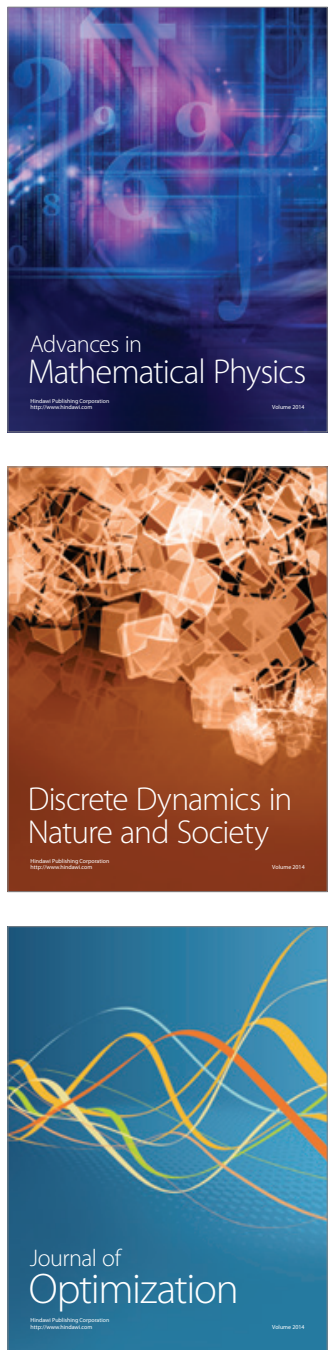